\title{
Efeitos dos Cogumelos Agaricus Blazei e Ganoderma Lucidum sobre o Desenvolvimento Embrionário e Gestacional de Ratas Wistar
}

\section{Effects of Agaricus Blazei and Ganoderma Lucidum Mushrooms on Embryonic and Gestational Development of Wistar Rats}

\author{
Camila Figueira Mendes ${ }^{1}$ \\ Erika Leão Ajala Caetano ${ }^{1}$ \\ Isabella Ferreira Camargo ${ }^{1}$ \\ Angela Faustino Jozala ${ }^{1,2}$ \\ Denise Grotto ${ }^{1,2}$ \\ Marli Gerenutti ${ }^{3}$ \\ Yoko Oshima-Franco ${ }^{1}$
}

\section{RESUMO}

Objetivo: Avaliar os efeitos sobre o desenvolvimento embrionário e gestacional em ratas prenhas expostas diariamente aos cogumelos Agaricus blazei e Ganoderma lucidum. Metodologia: Após a confirmação de prenhez ( $\left.1^{\circ} \mathrm{dia}\right)$, os animais foram separados aleatoriamente em 5 grupos ( $n=6$, cada): controle salina (Cs), A. blazei $\left(\mathrm{Ab}_{1-8}, 1^{\circ}-8^{\circ} \mathrm{dia}\right), A$. blazei $\left(\mathrm{Ab}_{9-19}, 9^{\circ}-19^{\circ}\right.$ dia), G. lucidum $\left(\mathrm{Gl}_{1-8}, 1^{1}-8^{\circ} \mathrm{dia}\right)$ e G. lucidum $\left(\mathrm{GI}_{1-19,}, 9^{\circ}-19^{\circ}\right.$ dia). Os animais receberam diariamente, via gavagem, 1,5 $\mathrm{mL}$ de cada cogumelo $(100 \mathrm{mg} / \mathrm{kg}$ de pó liofilizado dissolvido em solução salina). No $20^{\circ}$ dia gestacional, as fêmeas foram eutanizadas para a coleta de sangue (análises bioquímicas e hematológicas); rins e placenta (análises histológicas); cornos uterinos (análises morfométricas e morfológicas dos fetos). Resultados: Efeitos deletérios no perfil reprodutivo e gestacional das ratas e alterações nos parâmetros hematológicos não foram observados, como também hepatotoxicidade ou nefrotoxicidade materna. No entanto, ocorreu uma redução plasmática de colesterol total e triglicérides. O G. lucidum aumentou as células produtoras de glicogênio na placenta no período anterior à implantação. Quanto à prole ocorreu uma diminuição significativa no peso dos fetos de ambos os grupos expostos aos cogumelos. Conclusão: A administração oral dos cogumelos Agaricus blazei e Ganoderma lucidum em ratas prenhas positivamente reduziu o colesterol total e triglicérides, revelando um efeito cardioprotetor. $\mathrm{O}$ uso desses cogumelos durante $\mathrm{O}$ período gestacional foi seguro, não tendo apresentado efeitos teratogênicos, levando a uma redução de peso da prole, que pode ser útil em ocorrências do diabetes mellitus gestacional.

\section{DESCRITORES}

Agaricus Blazei. Ganoderma Lucidum. Gestação. Teratogenicidade.

\begin{abstract}
Objective: To evaluate the effects on embryonic and gestational development in pregnant rats exposed daily to Agaricus blazei and Ganoderma lucidum mushrooms. Methodology: After the confirmation of pregnancy (1st day) the animals were randomly separated into 5 groups $(n=6$, each): saline control (Cs), $A$. blazei (Ab1-8, 1st - 8th day), A. blazei (Ab9-19, 9th - 19th day), G. Iucidum (Gl1-8, 1st - 8th day) and G. Iucidum (GI1-19, 9th - 19th day). The animals received daily, via gavage, $1.5 \mathrm{~mL}$ of each mushroom (100 mg/kg of lyophilized powder dissolved in saline solution). On the 20th gestational day, the females were euthanized for blood collection (biochemical and hematological analyses); kidneys and placenta (histological analyses); uterine horns (morphometric and morphological analyses of the fetuses). Results: No deleterious effects were observed in the reproductive and gestational profile of the rats, nor any changes in hematological parameters. No hepatotoxicity or maternal nephrotoxicity was observed, but there was a plasma reduction of total cholesterol and triglycerides. G. lucidum increased the glycogen-producing cells in the placenta in the period before implantation. As for the offspring, there was a significant decrease in the weight of the fetuses of both groups exposed to mushrooms. Conclusion: Oral administration of Agaricus blazei and Ganoderma lucidum mushrooms in pregnant rats positively reduced total cholesterol and triglycerides, revealing a cardioprotective effect. The use of these mushrooms during the gestational period was safe, had no teratogenic effects and led to a weight reduction of the offspring, which can be useful in occurrences of gestational Diabetes mellitus.
\end{abstract}

\section{DESCRIPTORS}

Agaricus Blazei. Ganoderma Lucidum. Gestation. Teratogenicity.

\footnotetext{
${ }^{1}$ Programa de Pós-Graduação em Ciências Farmacêuticas da Universidade de Sorocaba (PPGCF/UNISO), Sorocaba, São Paulo, Brasil. 2 Programa de Pós-Graduação em Processos Tecnológicos e Ambientais da Universidade de Sorocaba (PPGPTA/UNISO), Sorocaba, São Paulo, Brasil.
}

${ }^{3}$ Faculdade de Ciências Médicas da Pontifícia Universidade Católica de São Paulo (PUC-SP), Sorocaba, São Paulo, Brasil. 
$\mathrm{O}$ danos no processo reprodutivo podem ter como causas a genética, o meio ambiente ou, ainda, a associação entre os dois fatores, podendo levar à morte do concepto (aborto), às malformações, ao retardo do crescimento intrauterino e deficiências nas funções orgânicas como o retardo mental ${ }^{1}$. A placenta, caracterizada pela justaposição de tecidos maternos e fetais, tem por finalidade a troca fisiológica de nutrientes e oxigênio entre a mãe e o feto para o desenvolvimento do embrião, além de funções endócrinas, secretoras e protetoras ${ }^{2}$. A passagem de substâncias pela placenta depende de vários fatores, tais como, ligação às proteínas plasmáticas, solubilidade de lipídios e constante de ionização $(\mathrm{pK})^{3}$.

O desenvolvimento normal do embrião ou do feto depende de uma herança genética adequada e do meio ambiente intrauterino, que está sujeito a agressões de agentes infecciosos, da má nutrição materna, do tabagismo e do uso de outras drogas, da insuficiente irrigação placentária e das enfermidades maternas, como exemplos ${ }^{4}$. Uma vez que uma dieta de alta qualidade junto com adequada ingestão de macro e micronutrientes durante a gravidez é crucial para o estado saudável da mãe e do feto 5 , pode-se pensar nos cogumelos comestíveis, pois são considerados alimentos funcionais de elevada qualidade nutricional e utilizados em forma de chás ou cápsulas como preventivos de algumas doenças ${ }^{6}$.

Entretanto, igualmente importante é conhecer se uma ingestão de cogumelos durante a gravidez poderia afetar negativamente o desenvolvimento e produzir efeitos tóxicos, dentre eles, o teratogênico. Conceitua-se como teratógeno qualquer substância (agente químico, físico ou biológico) que, ao estar presente ao longo da vida do embrião ou do feto, pode ocasionar alterações estruturais (se ocorrer no período teratogênico) ou funcionais (período até o nascimento) na descendência ${ }^{7}$. Os mecanismos moleculares de ação mais comuns dos teratógenos são hiperacetilação, mas substâncias que causam desequilíbrio do colesterol, alteração do metabolismo, antagonismo do folato, desequilíbrio do ácido retinóico, desregulação endócrina, desregulação vascular e estresse oxidativo também podem ser responsáveis por causar a teratogênese ${ }^{8}$.

O cogumelo Agaricus blazei tem alta concentração de tocoferóis, compostos responsáveis pela sua atividade antioxidante ${ }^{9}$. O A. blazei contém a enzima antioxidante polifenol oxidase ${ }^{10}$. O cobre, o zinco e o selênio são metais presentes no cogumelo $A$. blazei e podem ser benéficos para a proteção contra a geração de radicais, uma vez que os minerais participam diretamente de processos de eliminação de radicais, além de estarem contidos na estrutura de enzimas antioxidantes. O cogumelo Ganoderma lucidum apresenta uma variedade de atividades biológicas, tais como: imunoestimulante, antimicrobiano, anti-inflamatório, anti-hiperglicêmico, proteção hepática e renal, efeitos antioxidantes e antivirais ${ }^{11}$. Os fenóis totais foram um dos componentes antioxidantes encontrados nos extratos de G. Iucidum ${ }^{12}$.

Tendo em vista a importância da nutrição materna para o desenvolvimento normal dos conceptos, o objetivo do trabalho foi estudar o perfil gestacional de ratas Wistar expostas aos cogumelos Agaricus blazei e Ganoderma lucidum durante os períodos de 
pré- e pós-implantação que correspondem ao $1^{\circ}$ ao $8^{\circ}$ e $9^{\circ}$ ao $19^{\circ}$ dias gestacionais, respectivamente.

\section{METODOLOGIA}

O projeto foi aprovado pela Comissão de Ética no Uso de Animais da Universidade de Sorocaba CEUA/UNISO Protocolo 089/2016.

Os cogumelos foram adquiridos comercialmente da Yuri Cogumelos (Sorocaba, SP, Brasil), cortados e armazenados em biofreezer a $-80^{\circ} \mathrm{C}$ por $24 \mathrm{~h}$. Posteriormente, o material congelado foi liofilizado (Módulo D Freezer Dryer, Thermo Electron Corp.), até a obtenção de $10 \%$ de massa seca. A amostra seca foi moída (moinho de facas e martelo marca Marconi ${ }^{\circledR}$ ), em seguida tamisada em malha 50 e 60 Mesh. O pó tamisado foi acondicionado hermeticamente em embalagens plásticas, sendo mantidas em dessecador até o momento do uso.

Os animais foram adquiridos do Instituto de Ciências Biomédicas da Universidade de São Paulo (São Paulo, SP, Brasil) e receberam certificação sanitária. Foram mantidos em laboratório climatizado e receberam ração e água ad libitum. Ratos Wistar, machos e fêmeas pesando em torno de 180 a $200 \mathrm{~g}$ foram alojados em número de três, 01 macho com 02 fêmeas, por um período de 12 horas, para acasalamento. Constatada a prenhez, as ratas foram divididas em 5 grupos de seis animais $(n=6)$ : Grupo CS: controle (solução salina). Grupo $A b_{1-8}$ : A. blazei $1^{\circ}$ ao $8^{\circ}$ dia, Grupo $\mathrm{Ab}_{9-19}$ : A. blazei $9^{\circ}$ ao $19^{\circ} \mathrm{dia}$, Grupo $\mathrm{GI}_{1-8}$ : G. lucidum $1^{\circ}$ ao $8^{\circ} \mathrm{dia}$, Grupo $\mathrm{Gl}_{1-19}$ : G. lucidum $9^{\circ}$ ao $19^{\circ}$ dia. Nos períodos es- tabelecidos acima, os animais receberam via gavagem $1,5 \mathrm{~mL}$ de $100 \mathrm{mg} / \mathrm{kg}$ de pó liofilizado solubilizado em solução salina. O peso dos animais foi acompanhado nos dias: $1^{\circ}, 4^{\circ}, 8^{\circ}$, $12^{\circ}, 16^{\circ}$ e $20^{\circ}$. No vigésimo dia de prenhez, as ratas foram anestesiadas com uma combinação ${ }^{13}$ quetamina $(100 \mathrm{mg} / \mathrm{kg}$, anestésico dissociativo) e cloridrato de xilazina $(6 \mathrm{mg} /$ $\mathrm{kg}$, sedativo, analgésico e relaxamento muscular), via intraperitoneal (i.p.), para a coleta de amostras biológicas. O sangue foi coletado (em tubo sem anticoagulante e em tudo com EDTA) por punção venosa, seguindo-se com a coleta dos cornos uterinos, retirada dos fetos, das placentas, reabsorções e ovários. Esses materiais biológicos foram colocados em sulfeto de amônio $1 \%$, por 10 minutos, para a contagem dos sítios iniciais de implantação e de reabsorções ${ }^{14}$. As placentas, fetos e ovários foram pesados e o número de corpos lúteos nos ovários contados através da observação em microscópio e os fetos analisados para a identificação de malformações externas e ósseas.

A função hepática materna foi avaliada pelas dosagens de aspartato aminotransferase (AST) e alanina aminotransferase (ALT); enquanto a função renal materna foi avaliada pelas dosagens sanguíneas de creatinina e ureia, utilizando-se kits comerciais adquiridos do laboratório Rochec (São Paulo, Brasil) e os resultados comparados aos do grupo salina. Em ambos os procedimentos seguiram-se as recomendações do fabricante e foram realizadas em equipamento automatizado (Cobas C111, Rochec, São Paulo, Brasil).

Os níveis de glicose, triglicérides, colesterol e HDL foram obtidos através do equipamento automático Cobas C111, utilizando 
kits comerciais (Roche ${ }^{c}$ São Paulo, Brasil). As análises foram realizadas de acordo com as instruções do fabricante.

A hemoglobina, o hematócrito, os glóbulos brancos (White blood cells - WBC), a contagem total de eritrócitos (red blood ceIls - RBC) e o número de plaquetas (platelets, PLT) foram analisados através do equipamento automático Hematologia XS 1000i WAS, Roche ${ }^{\circledR}$.

As placentas e os rins foram fixados em formalina $10 \%$ tamponada, em seguida, incluídos em parafina e seccionados para obtenção dos cortes histológicos, os quais foram corados com hematoxilina e eosina para posterior análise histológica. Também foram realizadas análises nos fetos. Os mesmos foram anestesiados e eutanasiados com halotano, divididos em dois grupos de análises: (1) análises morfológicas e morfométricas externas (fixados em líquido de Bodian - foram realizadas as seguintes medidas: antero-posterior do crânio, látero-lateral do crânio, antero-posterior do tórax, látero-lateral do tórax, crânio-caudal, cauda; (2) análise óssea (procedimentos de evisceração, diafanização e coloração com Alizarin red S plus Alcian blue - contagem de ossos e avaliação de agenesia ósseas de crânio, costelas, vértebras e membros ${ }^{15,16}$.

Os resultados foram expressos como média \pm desvio padrão $(M \pm D P)$ e analisados estatisticamente por meio de ANOVA, seguida do teste Tukey-Kramer, com nível de significância entre 1 e $5 \%$.

\section{RESULTADOS}

A Tabela 1 apresenta os dados de variação de peso e desempenho reprodutivo de ratas expostas aos cogumelos durante os períodos pré- e pós-implantacional. $\mathrm{O}$ intervalo da variação de peso do grupo Cs foi de 35,21-160,29 g durante todo o período. Observou-se um aumento significativo no número de fetos nascidos vivos no grupo que recebeu $A$. blazei antes da implantação $\left(A b_{1-8}\right)$

Tabela 1. Ganho de peso materno e desempenho reprodutivo

\begin{tabular}{l|c|c|c|c|c}
\hline \multirow{2}{*}{} & $\mathrm{Cs}$ & $\mathrm{Ab}_{1-8}$ & $\mathrm{Ab}_{9-19}$ & $\mathrm{Gl}_{1-8}$ & $\mathrm{Gl}_{9-19}$ \\
\cline { 2 - 6 } & \multicolumn{5}{|c}{$\mathrm{M} \pm \mathrm{DP}$} \\
\hline$\Delta$ Peso corporal $\left(1^{\circ}-20^{\circ} \mathrm{dg}\right)$ & $97,8 \pm 62,5$ & $114,5 \pm 10,6$ & $103,5 \pm 17,3$ & $120,0 \pm 8,3$ & $100,8 \pm 10,9$ \\
\hline Corpos Lúteos (n) & $12,3 \pm 1,7$ & $13,8 \pm 1,7$ & $11,5 \pm 1,5$ & $13,3 \pm 2,5$ & $11,5 \pm 1,6$ \\
\hline Sítios implantação (n) & $10,6 \pm 3,1$ & $13,5 \pm 1,6$ & $11,3 \pm 1,8$ & $13,3 \pm 2,5$ & $10,5 \pm 2,4$ \\
\hline Perdas pós- implantação (\%) & 6,8 & 3,7 & 2,9 & 2,5 & 4,7 \\
\hline Total reabsorção (n) & $0,7 \pm 0,7$ & $0,5 \pm 1,2$ & $0,3 \pm 0,5$ & $0,3 \pm 0,5$ & $0,5 \pm 0,6$ \\
\hline Fetos mortos (n) & $0,3 \pm 0,5$ & - & - & $0,2 \pm 0,4$ & $0,2 \pm 0,4$ \\
\hline Fetos vivos (n) & $9,6 \pm 2,4$ & $13,0 \pm 1,6^{*}$ & $11,0 \pm 1,8$ & $12,8 \pm 2,6$ & $9,8 \pm 1,7$ \\
\hline
\end{tabular}

Cs (Controle - solução salina 0,9\%), Ab ${ }_{1-8}$ (Agaricus blazei antes da implantação), $\mathrm{Ab}_{9-19}$ (Agaricus blazei depois da implantação), $\mathrm{GI}_{1-8}$ (Ganoderma lucidum antes da implantação), $\mathrm{GI}_{9-19}$ (Ganoderma lucidum depois da implantação). ${ }^{*} \mathrm{p}<0.05$ em comparação ao grupo Controle salina (Cs). 
comparado ao controle. Ocorreu também uma redução na média da relação feto/placenta, de 6,0 para 5,0 .

A Figura 1 apresenta os dados do perfil hematológico de ratas expostas aos cogumelos durante os períodos pré e pós implantacional. A análise estatística dos dados de perfil hematológico materno não demonstrou nenhuma significância nos parâmetros WBC, RBC, $\mathrm{Hb}$, Ht e PLT dos grupos comparativamente ao grupo controle CS.

Os cogumelos Agaricus blazei $(A b)$ e Ganoderma lucidum (GI), antes (1-8) e depois (9-19) do período implantacional, sendo série branca: WBC (White blood Cells); série vermelha: RBC (Red Blood Cells), Hb, Hemoglobina, Ht, hematócrito; e série plaquetária: plaquetas. As unidades estão expressas no título do eixo Y.
A Figura 2 ilustra o perfil bioquímico materno. Não foi observada nenhuma diferença estatística no perfil hepático, no perfil renal, nem nos marcadores glicêmicos e colesterolêmicos, comparados ao controle, demonstrando que a exposição aos cogumelos não influenciou o metabolismo materno durante o período gestacional.

Os cogumelos A. blazei e G. lucidum. Função hepática: (A) ALT (U/L), (B)AST (U/L). Função renal: (C) Ureia (mg/dL), (D) Creatinina $(\mathrm{mg} / \mathrm{dL}),(E)$ Albumina $(\mathrm{g} / \mathrm{dL})$. Disfunções lipidêmicas: (F) Colesterol (mg/dL), (G) HDL $(\mathrm{mg} / \mathrm{dL})$. Disfunções relacionadas ao açúcar: (H) Glicose (mg/dL), (I) Triglicérides ( $\mathrm{mg} / \mathrm{dL}$ ). Função pancreática: (J) Lipase (U/L), (K) Insulina (ng/mL). * $p<0.05$ em comparação ao grupo Controle salina (Cs). Grupos experimentais são apresentados na seguinte sequência: Cs

Figura 1. Perfil hematológico e bioquímico de ratas expostas aos cogumelos durante os períodos pré e pós-implantacional

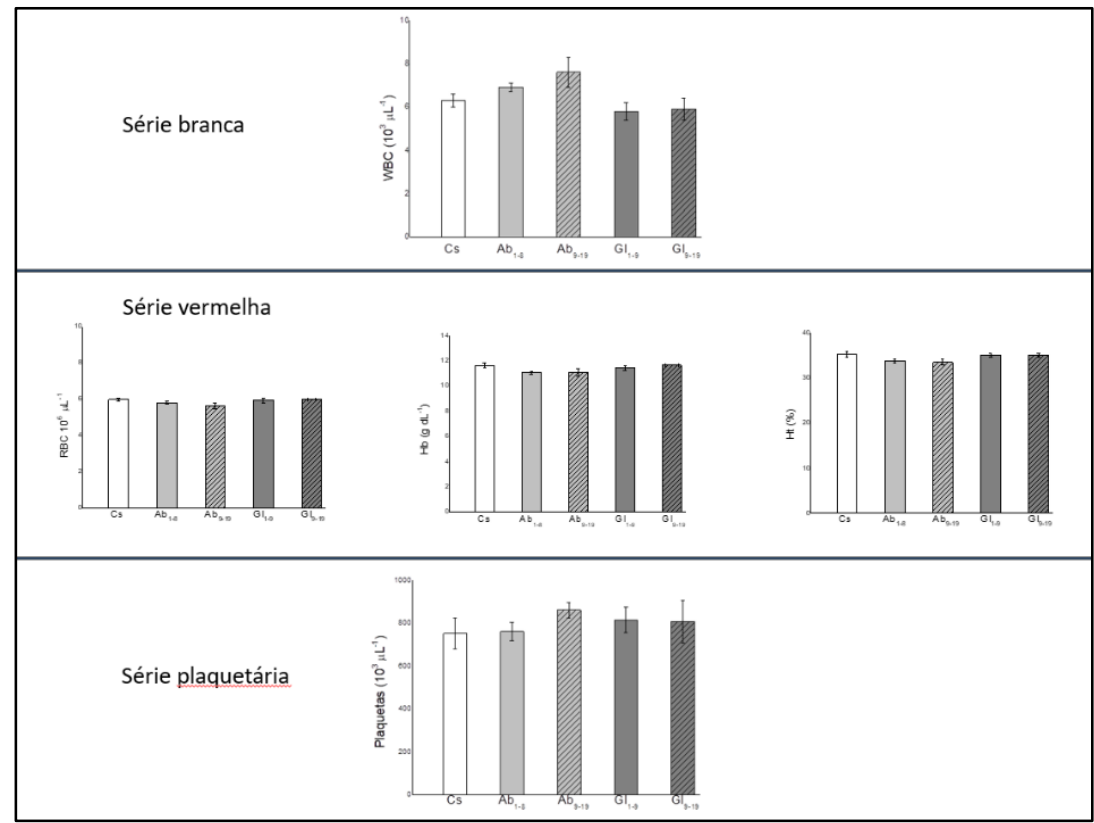

Os cogumelos Agaricus blazei (Ab) e Ganoderma lucidum (GI), antes (1-8) e depois (9-19) do período implantacional, sendo série branca: WBC (White blood Cells); série vermelha: RBC (Red Blood Cells), Hb, Hemoglobina, Ht, 
Figura 2. Perfil bioquímico frente à exposição materna durante a prenhez

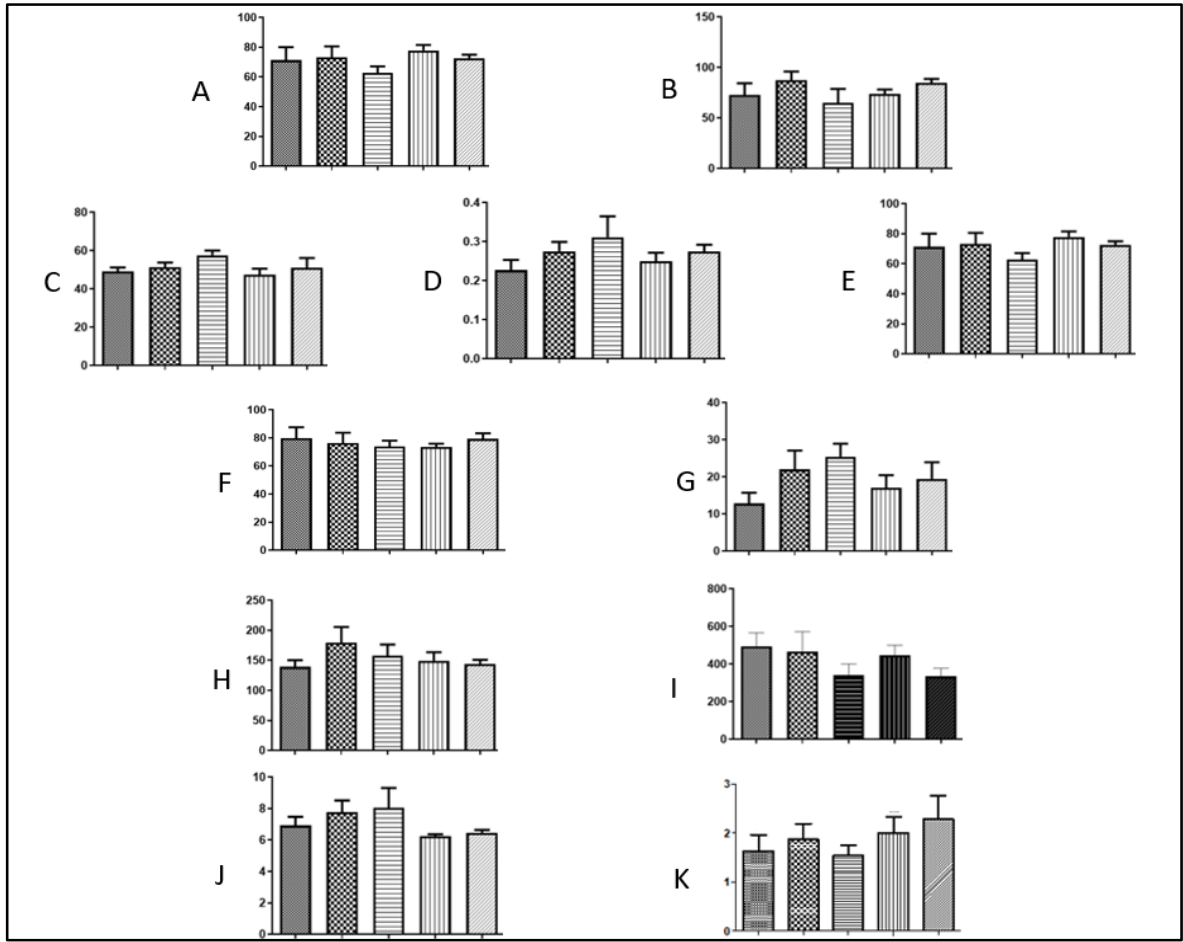

Os cogumelos A. blazei e G. lucidum. Função hepática: (A) ALT (U/L), (B) AST (U/L). Função renal: (C) Ureia (mg/dL), (D) Creatinina (mg/dL), (E) Albumina (g/dL). Disfunções lipidêmicas: $(F)$ Colesterol ( $\mathrm{mg} / \mathrm{dL}),(\mathrm{G}) \mathrm{HDL}$ ( $\mathrm{mg} / \mathrm{dL})$. Disfunções relacionadas ao açúcar: (H) Glicose ( $\mathrm{mg} / \mathrm{dL})$, (I) Triglicérides $(\mathrm{mg} / \mathrm{dL})$. Função pancreática: (J) Lipase (U/L), (K) Insulina ( $\mathrm{ng} / \mathrm{mL})$. ${ }^{*} \mathrm{p}<0.05$ em comparação ao grupo Controle salina (Cs). Grupos experimentais são apresentados na seguinte sequência: Cs (Controle salina 0,9\%); $\mathbf{A b}_{1-8}$ (Agaricus blazei antes da implantação); $\mathbf{A} \mathbf{b}_{9-19}$ (Agaricus blazei depois da implantação); Gl $\mathbf{1}_{1-8}$ (Ganoderma lucidum antes da implantação); e Glg-19 (Ganoderma lucidum depois da implantação).

Tabela 2. Desempenho gestacional de ratas expostas aos cogumelos A. blazei e G. lucidum durante os períodos pré- e pós-implantacional

\begin{tabular}{|c|c|c|c|c|c|}
\hline & Cs & $\mathrm{Ab}_{1-8}$ & $\mathrm{Ab}_{9-19}$ & $\mathrm{Gl}_{1-8}$ & $\mathrm{GI}_{9-19}$ \\
\hline Corpos lúteos & $12,3 \pm 1,6$ & $13,8 \pm 1,6$ & $11,5 \pm 1,5$ & $13,3 \pm 1,6$ & $11,5 \pm 1,6$ \\
\hline Sítios de implantação & $12,3 \pm 1,6$ & $13,8 \pm 1,6$ & $11,5 \pm 1,5$ & $13,3 \pm 1,6$ & $11,5 \pm 1,6$ \\
\hline Reabsorções precoces & $0,7 \pm 0,7$ & $0,8 \pm 0,7$ & $0,3 \pm 0,5$ & $0,3 \pm 0,66$ & $0,5 \pm 0,6$ \\
\hline Reabsorções tardias & - & - & - & - & - \\
\hline Total de reabsorções & $0,7 \pm 0,7$ & $0,8 \pm 0,7$ & $0,8 \pm 1,3$ & $0,3 \pm 0,7$ & $0,5 \pm 0,6$ \\
\hline Fetos mortos & - & - & - & - & $0,2 \pm 0,4$ \\
\hline Fetos vivos & $11,3 \pm 1,3$ & $13,0 \pm 1,3$ & $11,0 \pm 1,79$ & $12,8 \pm 1,3$ & $10,8 \pm 1,8$ \\
\hline
\end{tabular}

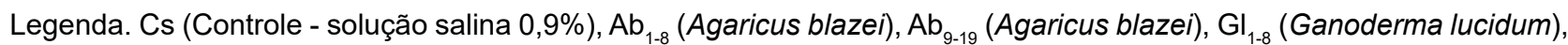
$\mathrm{Gl}_{9-19}$ (Ganoderma lucidum). * $\mathrm{p}<0.05$ em comparação ao grupo Controle (Cs). 
Figura 3. Fotomicrografia de corte histológico de placentas de ratas Wistar

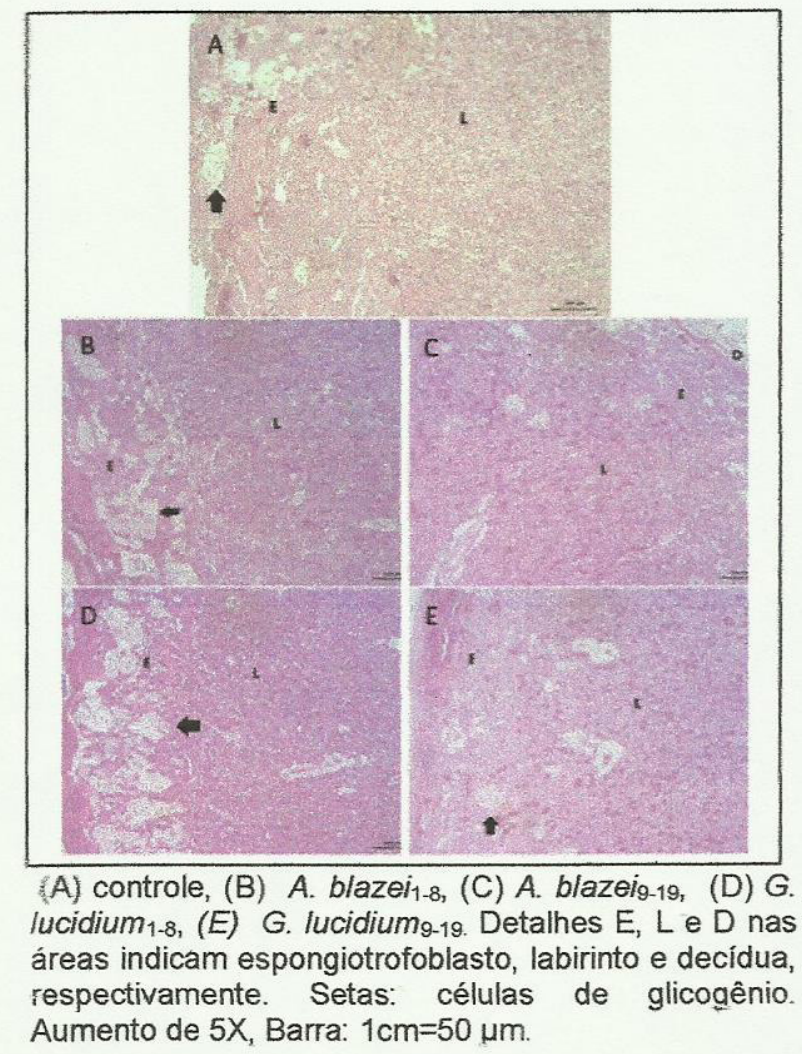

(Controle salina 0,9\%); $\mathrm{Ab}_{1-8}$ (Agaricus blazei antes da implantação); $\mathrm{Ab}_{9-19}$ (Agaricus blazei depois da implantação); $\mathrm{GI}_{1-8}$ (Ganoderma lucidum antes da implantação); e $\mathrm{Gl}_{9-19}$ (Ganoderma lucidum depois da implantação).

A Tabela 2 ilustra os parâmetros de desempenho gestacional das ratas expostas aos cogumelos nos períodos pré- e pós-implantacional. Pode-se observar que nenhuma diferença foi encontrada comparando os grupos, sugerindo que os cogumelos não interferiram no desempenho gestacional. Analisando estatisticamente os índices gestacionais não houve diferença significativa entre o tratamento com os cogumelos nos períodos de pré- e pós-implantação quando comparado ao controle.
A avaliação histológica da estrutura morfológica da placenta de ratas expostas aos cogumelos durante as fases de pré- e pós-implantação do concepto está demonstrada na Figura 3. As estruturas placentárias E, L e $D$ destacadas nas fotografias correspondem ao espongiotrofoblasto, labirinto e decídua, respectivamente. As setas indicam as células de glicogênio. A partir da análise microscópica do tecido foi possível constatar que a estrutura placentária não sofreu nenhuma mudança histológica após a exposição aos cogumelos nos período pré e pós-implantação, quando comparado ao grupo controle.

A Figura 4 ilustra as áreas corticais renais de ratas expostas aos cogumelos durante os períodos pré- e pós-implantação 
Figura 4. Fotomicrografias de cortes longitudinais da região do córtex renal de ratas Wistar

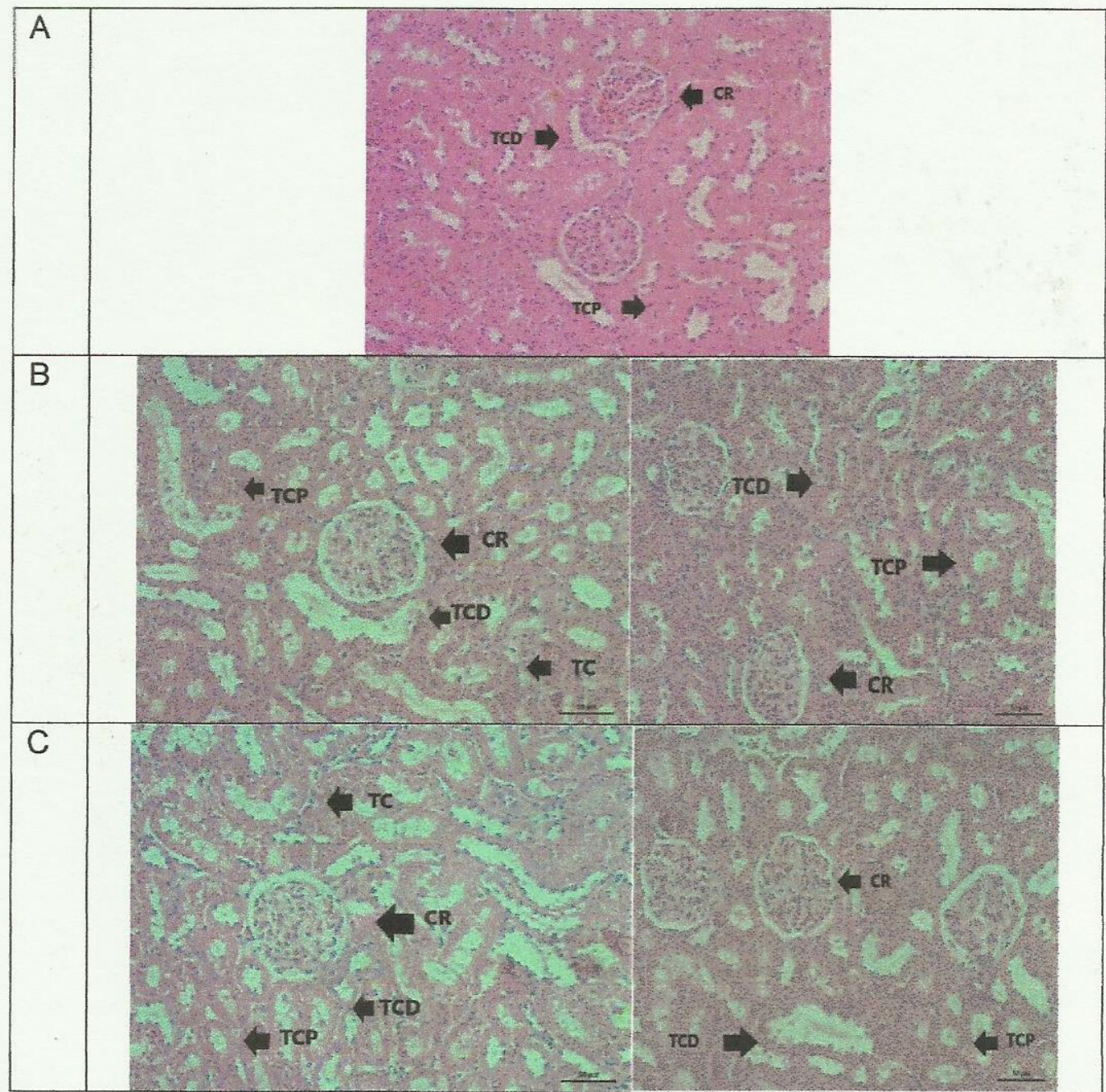

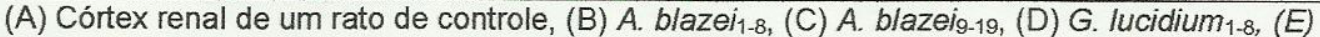
$G$. lucidium 9-19. TCD, Túbulo contorcido distal. TCP, Túbulo contorcido proximal. CR. Corpúsculo renal. Objetiva de 20X. Barra: $1 \mathrm{~cm}=50 \mu \mathrm{m}$

do concepto. Observou-se que as estruturas morfológicas dos glomérulos maternos não sofreram alterações nos períodos considerados frente ao tratamento com ambos os cogumelos, comparativamente ao controle.

A toxicologia fetal foi avaliada por parâmetros apresentados na Figura 5, como: (A) peso fetal ao nascimento, (B) peso da placenta e morfometria fetal (C-G). Ocorreu uma diminuição significativa no peso dos fetos de ambos os grupos expostos aos cogumelos, antes e depois da implantação, comparativamente ao controle (Fig. 5A).

(A) peso fetal (em g) ao nascimento. (B) peso da placenta (em g). (C) morfometria fetal crânio látero/lateral. (D) morfometria fetal crânio antero/posterior. (E) morfometrial fetal crânio/caudal. (F) morfometria fetal tó- 
Figura 5. Toxicologia fetal

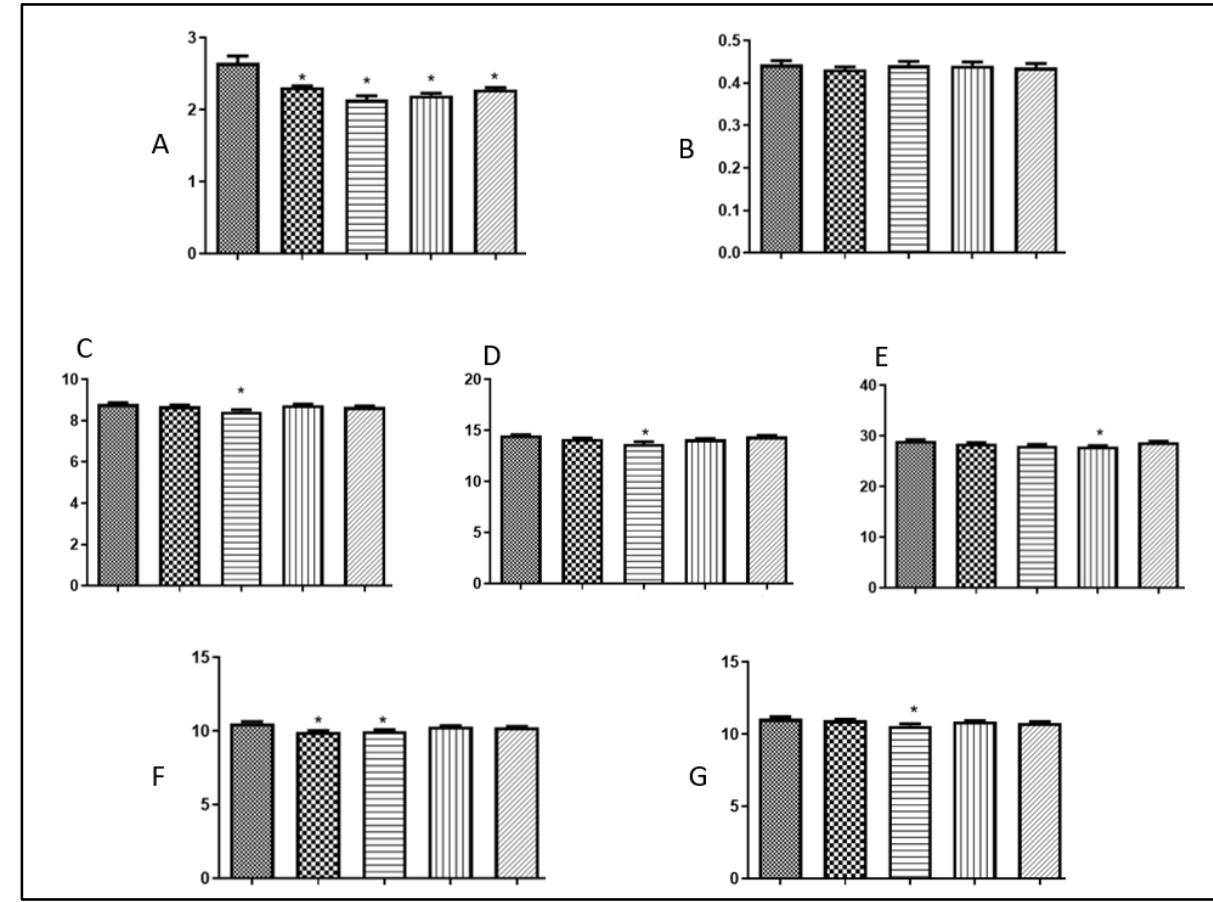

(A) peso fetal (em g) ao nascimento. (B) peso da placenta (em g). (C) morfometria fetal crânio látero/lateral. (D) morfometria fetal crânio antero/posterior. (E) morfometrial fetal crânio/caudal. (F) morfometria fetal tórax látero/lateral. (G) morfometria fetal tórax antero/posterior. Grupos experimentais: $\mathrm{Cs}$ (Controle salina $0,9 \%$ ); $* \mathrm{Ab}_{1-8}$ (Agaricus blazei antes da implantação); $\sqsupseteq$ Ab9-19 (Agaricus blazei depois da implantação); IIIIII $\mathbf{G l}_{1-8}$ (Ganoderma lucidum antes da implantação); e Ver $\mathbf{I G I}_{1-19}$ (Ganoderma lucidum depois da implantação). ${ }^{*} p<0.05$ em comparação ao grupo Controle salina (Cs); Anova, seguida de Tukey-Kramer.

rax látero/lateral. (G) morfometria fetal tórax antero/posterior. Grupos experimentais: Cs (Controle salina 0,9\%); $\approx \approx \mathrm{Ab}_{1-8}$ (Agaricus blazei antes da implantação); $\mathrm{Ab}_{9-19}$ (Agaricus blazei depois da implantação); IIIIIIG| $\left.\right|_{1-8}$ (Ganoderma lucidum antes da implantação); e vira $\mathrm{GI}_{1-19}$ (Ganoderma lucidum depois da implantação). * $p<0.05$ em comparação ao grupo Controle salina (Cs); Anova, seguida de Tukey-Kramer.

Verificou-se que as placentas não sofreram alteração de peso durante todo o período experimental (Fig. 5B). Entretanto, estatisticamente ocorreu uma redução nos seguintes parâmetros: na medida crânio antero-posterior no grupo $A$. blazei depois da implantação (Fig. 5D, $A b_{1-8}$ ); na medida crânio-caudal antes da implantação (Fig. 5E, $\mathrm{GI}_{1-8}$ ); na morfometria tórax latero-lateral nas ratas que receberam $A$. blazei antes e após a implantação (Fig. 5F); e, na morfometria fetal tórax antero/posterior o grupo A. blazei antes da implantação (Fig. 5G, Ab ${ }_{1-8}$ ).

\section{DISCUSSÃO}

A segurança dos alimentos durante a fase gestacional é um fator importante na 
redução dos riscos de teratogenicidade e a busca por alimentos que garantam os nutrientes necessários para o desenvolvimento normal deve ser continuamente investigada. Os cogumelos comestíveis representam uma alternativa tendo em vista seu elevado potencial nutricional, cuja composição varia conforme o tipo, sendo $19-35 \%$ proteínas, ricos em aminoácidos essenciais como lisina, valina e leucina, possuem 72 a $85 \%$ de gorduras poli-insaturadas, baixos índices de carboidratos, ricos em fibras e possuem vitaminas como tiamina, riboflavina, niacina, biotina, vitamina $C$, vitaminas do complexo $B$ e minerais ${ }^{17,18}$. Neste estudo avaliaram-se os efeitos da administração oral dos cogumelos Agaricus blazei e Ganoderma lucidum nos períodos pré- e pós-implantacional de ratas prenhas.

O primeiro parâmetro apresentado foi a variação no ganho ou na perda de peso. Essas medidas, seja de peso corporal ou de massa relativa de órgãos, são importantes para o monitoramento da saúde materna e desenvolvimento fetal e são utilizadas em diferentes áreas da toxicologia, como na toxicologia médica, ambiental e alimentar para avaliar o efeito de certas substâncias, durante a gestação ${ }^{19-22}$. Comparativamente à variação que sofreu o grupo controle $\mathrm{Cs}$, todos os grupos $\left(A b_{1-8}, A b_{9-19}, G_{1-8}, G I_{1-19}\right)$ oscilaram na mesma faixa, ou seja, nos limites considerados normais. Estudos pré-clínicos, realizado anteriormente por Viroel e Gerenu$\mathrm{tti}^{23}$, com ratas Wistar prenhas expostas ao pó liofilizado do cogumelo $\mathrm{G}$. lucidum (dose única de $40 \mathrm{mg} / \mathrm{kg}$, IV), no oitavo dia de gestação 22 demonstraram índices de baixo peso, embora os autores não tenham sugerido uma explicação metabólica plausível.
Conforme demonstra a Tabela 1, no grupo $\left(A b_{1-8}\right)$ observou-se um aumento de fetos nascidos vivos. No trabalho de Laurino et al. ${ }^{24}$, as ratas Wistar prenhas, expostas ao pó liofilizado do cogumelo Lentinus edodes (100 mg/kg/dia), antes e após a implantação fetal não foram observadas alterações no número de fetos vivos por mãe entre os grupos estudados e o controle negativo, divergindo ao resultado encontrado nesta pesquisa.

Observa-se a ausência de significância no peso dos ovários e da placenta, além da diminuição significativa do peso fetal, independente do período gestacional (Fig.1). Ao se analisar a relação feto/placenta, observou-se uma diminuição, em média, 6,0 para 5,0 no grupo $A$. blazei e $G$. lucidum, mostrando que na presença de ambos os cogumelos, o crescimento fetal parece ter sido mais vulnerável do que o crescimento placentário. Contudo, sabe-se que a restrição do crescimento placentário é um fator determinante para a restrição do crescimento fetal (peso e comprimento), visto que isso possibilita a diminuição de acessos a nutrientes e oxigênio pela barreira materno - fetal ${ }^{25}$. Por outro lado, não foi identificado neste estudo, restrição no crescimento placentário, restando assim, a necessidade de estudos a cerca da organização histológica placentária, a qual possa estar interferindo no desenvolvimento fetal.

A exposição do organismo materno a ambos os cogumelos, nos períodos gestacionais considerados, mostra que não ocorreu interferência no desempenho gestacional. De modo geral, as perdas embrionárias foram em média 2 ou 3 vezes menores nos grupos tratados, quando comparados ao controle, porém, estatisticamente não existiu diferen- 
ça significativa, podendo-se apenas inferir uma tendência diminuída na quantidade de reabsorções e fetos mortos nos grupos tratados $(p>0,05)$. Este bom resultado pode estar relacionado à ação imunoestimuladora, principalmente, já atribuída à G. lucidum ${ }^{26}$ e corroborado pelo estudo de Bitzer et al. ${ }^{27}$, que avaliaram a exposição a glicoproteínas (proteínas que contem cadeias de oligossacarides, glicanas, covalentemente ligadas a cadeias laterais de polipeptídeos, por meio de um processo conhecido como glicosilação), produzidas pelo cogumelo Dacryopinax spathularia sobre o desempenho reprodutivo e o desenvolvimento embrionário de ratos, em que não se observou alterações sobre a sobrevivência, o peso corporal, o crescimento uterino e a morfologia fetal.

Durante a gestação podem ocorrer alterações fisiológicas como o aumento da diurese e do fluxo plasmático renal que modificam o processo farmacocinético dos medicamentos. Essas alterações, por sua vez, poderão interferir na intensidade e duração do efeito dos fármacos, sendo aconselhável monitorar os níveis plasmáticos para evitar efeitos danosos ao organismo da mãe e também do feto ${ }^{28}$. A intensa alteração hormonal pode ter efeitos sobre os parâmetros hematológicos e bioquímicos ${ }^{29}$, promovendo uma hemodiluição sanguínea e reduzindo os níveis de ureia e creatinina, os quais não foram observados neste trabalho. A ocorrência de nefrotoxicidade com cogumelos cosmetíveis é rara, porém, já ocorreu com a Amanita vaginata var punctat a (A. punctata $)^{30}$, o que valoriza a preocupação de avaliar este parâmetro em nosso estudo.

Os valores obtidos para AST e ALT para os grupos $A b_{1-8}$ (Agaricus blazei), $A b_{9-19}$
(Agaricus blazei), $\mathrm{GI}_{1-8}$ (Ganoderma lucidum), $\mathrm{GI}_{9-19}$ (Ganoderma lucidum) não foram estatisticamente diferentes do grupo Cs, evidenciando que os cogumelos administrados durante a gestação não induziram hepatoxicidade. Esses resultados são corroborados pelo que se conhece em relação aos cogumelos comestíveis, que são protetores hepáticos, incluindo Ganoderma lucidum que foi um dos mais estudados cogumelos, explicados pela presença de metabólitos secundários tais como compostos fenólicos, terpenos, esteroides, além de componentes essenciais da parede celular como polissacárides, $\beta$-glucanas e proteínas ${ }^{31}$. As dosagens bioquímicas de colesterol total, HDL-colesterol e triglicérides realizadas em soros de ratas (Tabela 3 ) não apresentaram aumento significativo de colesterol total e triglicérides. Pelo contrário, ocorreu uma diminuição, mesmo que não apresentando significância, o que pode nos direcionar a um possível efeito protetor contra doenças cardiovasculares. Sabe-se que o aumento do nível do colesterol total e triglicérides são fatores importantes na determinação de predisposição a doenças cardiovasculares ${ }^{32,33} \mathrm{e}$ que cogumelos podem combatê-las pelo baixo conteúdo em calorias, gorduras e sódio, além de serem livres de colesterol ${ }^{18}$. Nesta pesquisa observou-se aumentos pontuais nos níveis de colesterol HDL e diminuição dos níveis de colesterol total e triglicérides, corroborando a outros estudos ${ }^{34,35}$. Além disso, os hipolipidêmicos atuam como agentes protetores do infarto do miocárdio e doenças coronarianas ${ }^{36}$, uma grande promessa a qualquer candidato a este efeito, como os cogumelos comestíveis.

Ademais, não ocorreu diferença significativa dos níveis de insulina e glicose, 
quando comparados ao controle, apenas, uma tendência de aumento, que segundo alguns autores ${ }^{37}$, representariam flutuações fisiológicas. Por outro lado, estudos realizados $^{38-41}$ demonstraram a ação hipoglicemiante quando da administração dos cogumelos, não observado em nossos estudos e que tornam sugestivo o consumo desses cogumelos em condições de diabetes mellitus gestacional.

Observou-se que o grupo tratado com A. blazei, no período organogenético (9-19), apresentou crescimento crânio-caudal, caudal e crânio (antero-posterior) significativamente diminuídos, quando comparado ao grupo controle. Similarmente, os animais pertencentes ao grupo G. lucidum (1-8) apresentaram um menor crescimento, quando comparados ao grupo controle. No trabalho de Viroel e Gerenutti ${ }^{23}$, os fetos das ratas expostas ao $\mathrm{G}$. lucidum mostraram maiores medidas fetais de cabeça, tórax, crânio-caudal e cauda, enquanto as ratas Wistar prenhas expostas ao pó liofilizado reconstituído de G. lucidum (dose única de $40 \mathrm{mg} / \mathrm{kg}$, via gavagem) no oitavo dia de gestação, mostrou efeitos protetores sobre os fetos. Em outro trabalho de Valko et al. ${ }^{38}$, os fetos das ratas expostas ao G. lucidum mostraram maiores medidas fetais de cabeça, tórax, crânio-caudal e cauda, este efeito genotóxico ocorreu devido ao tempo de exposição ao cogumelo. Pode-se inferir que a maior exposição ao $\mathrm{G}$. lucidum pode causar efeitos genotóxicos, portanto, o tempo de exposição deve ser considerado.

Os resultados de malformações externas, ósseas, craniana, de costelas, metacarpos e vértebras caudais não apresentaram relevância estatística e, com base nos dados obtidos, não se observou efeito teratogênico induzido pelo uso de A. blazei e G. lucidum. Estes resultados corroboram com o estudo de Li et al. ${ }^{42}$, em que ratas grávidas receberam um extrato aquoso de $\mathrm{A}$. bisporus por 15 dias, o A. blazei e A. bisporus, que pertencem ao mesmo gênero e possuem grau de similaridade genética de $3 \%{ }^{43}$.

A partir da análise microscópica da Figura 3 pode-se observar (na indicação da seta) que ocorreu um aumento na quantidade de células produtoras de glicogênio no tecido placentário, no tratamento com G. lucidum antes da implantação do concepto. Portanto, este cogumelo pode auxiliar na manutenção do concepto durante o desenvolvimento gestacional, tendo em vista que em resposta à implantação do blastocisto, o endométrio materno sofre uma reação decidual na qual as células do estroma decidual da placenta acumulam glicogênio e nutrientes e aumentam a função secretora para dar suporte ao embrião inicial ${ }^{44}$.

Além disso, a teratogenicidade pode estar relacionada a variantes genéticas, materna e fetal, que afetam a farmacocinética (absorção, distribuição, metabolismo, excreção, transporte placentário) e a ligação de um agente ao receptor ${ }^{45}$. No presente trabalho, não foram detectadas alterações significativas no tecido renal materno e, portanto, pode-se considerar que não ocorreu alteração na filtração renal.

\section{CONCLUSÃO}

A administração oral dos cogumelos Agaricus blazei e Ganoderma lucidum em ra- 
tas prenhas positivamente reduziu o colesterol total e triglicérides, revelando um efeito cardioprotetor. $\mathrm{O}$ uso desses cogumelos durante $\mathrm{O}$ período gestacional foi seguro na gestação de ratas, não apresentando efeitos teratogênicos e levando a uma redução de peso da prole, que pode ser útil em ocorrências de diabetes mellitus gestacional.

\section{AGRADECIMENTOS}

Taxa Capes/Prosuc; Fapesp (Projeto 15/24566-9); Finep/01.10.0659.00.

\section{REFERÊNCIAS}

1. Schüller-Faccini L, Schvartzman L, Cecchin C. Teratogênese humana e o SIAT. In: Sanseverino MT, Spritzer D, Schüller-Faccini L, eds. Manual de teratogênese. Porto Alegre: Editora da Universidade, 2011.

2. Carter AM, Enders AC. Placentation in mammals: definitive placenta, yolk sac and paraplacenta. Therigenology 2016; 86(1):278-87.

3. Werler MM. Congenital malformations and consequential epidemiology. Curr. Epidemiol. Rep. 2014; 2(1):8-12.

4. Aquino LA. Acompanhamento do crescimento normal. Rev. Ped. SOPERJ. 2011; 12(supl 1):15-20.

5. Danielewicz $H$, Myszczyszyn $G$, Devinska $A$, Boznanskia, Boznański A, Hirnle L. Diet in pregnancymore than food. Eur. J. Pediatr. 2017; 176(12):1573-79.

6. Furlani RPZ, Godoy HT. Valor nutricional de cogumelos comestíveis: uma revisão. Rev. Inst. Adolfo Lutz 2005 64(2):149-154

7. Dicke JM. Teratology: principles and practice. Med Clin North Am. 1989; 73(3):567-82.

8. Giavini E, Menegola E. Biomarkers of teratogenesis: suggestions from animal studies. Reprod. Toxicol. 2012; 34(2):180-185

9. Tsai S, Tsai H, Mau J. Antioxidant properties of Agaricus blazei, Agrocybe cylindracea and Boletus edulis. LWTFood Sci. Technol. 2007; 40(8):1392-1402.

10. Hashimoto S, Akanuma AM, Motoi M, Imai N. Effect of culture conditions on chemical composition and biological activities of Agaricus braziliensis. Int. J. Med. Mushrooms 2006; 8(4):329-342.

11. Zhu LN, Lu X, Tang Q, Liu Y, Zhou S, Yang Y, et al. Isolation, purification, and immunological activities of a low-molecular-weight polysaccharide from the Lingzhi or Reishi medicinal mushroom Ganoderma lucidum (Higher Basidiomycetes). Int. J. Med. Mushrooms 2013; 15(4):407-414

12. Mau JL, Lin HC, Chen CC. Antioxidant properties of

several medicinal mushrooms. J. Agr. Food Chem. 2002; 50(21):6072-6077.

13. Haskins SC, Patz JD, Farver TB. Xylazine and xylazineketamine in dogs. Am. J. Vet. Res. 1986; 47(3):636-641.

14. Salewsky E. Farbemethode zum markroskopinhennachweis von implantatconsstellen am uterus der ratter naunyn schumuderbergs. Arch. Pharmacol. 1964; 247(1):367-372.

15. Esteves-Pedro NM, Borim T, Nazato VS, Silva MG, Lopes PS, dos Santos MG, et al. In vitro and in vivo safety evaluation of Dipteryx alata Vogel extract. BMC Complement. Altern. Med. 2012; 12(9):1-9.

16. Randazzo-Moura $P$, Silva MG, Oshima-Franco $Y$ Groppo FC, Gerenutti M. The effect of aqueous extract of Cecropia glazioui Snethlage (Embauba) in the rat fetal development. Chin. Med. 2011; 2(3):115-119.

17. Kalac P. Chemical composition and nutritional value of European species of wild growing mushrooms: A review. Food Chem. 2009; 113(1):9-16.

18. Rahi DK, Malik D. Diversity of mushrooms and their metabolites of nutraceutical and therapeutic significance. J. Mycol. 2016; 2016:Article ID 7654123, 18 pages.

19. Bhaskar R, Mohanty B. Pesticides in mixture disrupt metabolic regulation: in silico and in vivo analysis of cumulative toxicity of mancozeb and imidacloprid on body weight of mice. Gen. Comp. Endocrinol. 2014; 205:226-234

20. Lu L, Fan Y, Yao W, Xie W, Guo J, Yan Y, et al. Safety assessment of the fermented Phylloporia ribis (Lonicera japonica Thunb.) mycelia by oral acute toxicity study in mice and 90-day feeding study in rats. Food Chem. Toxicol. 2014; 69:18-24.

21. Mukerji P, Rae JC, Buck RC, O'Connor JC. Oral repeated-dose systemic and reproductive toxicity of $6: 2$ fluorotelomer alcohol in mice. Toxicol. Rep. 2015; 2:130143.

22. Olson JA, Schwartz JA, Hahka D, Nguyen N, Bunch $\mathrm{T}$, Jensen GM, et al. Toxicity and efficacy differences 
between liposomal amphotericin B formulations in uninfected and Aspergillus fumigatus infected mice. Med. Mycol. J. 2015; 53(2):107-118.

23. Viroel FJM, Gerenutti M. Estudos pré-clínicos do cogumelo medicinal Ganoderma lucidum (Higher Basidiomycetes): evidências no diabetes mellitus gestacional. Repositório do Programa de Pós Graduação em Ciências Farmacêuticas, Universidade de Sorocaba, 2018.

24. Laurino LF, Viroel FJM, Caetano E, Spim S, Pickler TB, Rosa-Castro RM, et al. Lentinus edodes exposure before and after fetus implantation: materno-fetal development in rats with gestational diabetes mellitus. Nutrients 2019; 11(11):2720.

25. Schafer ZT, Parrish AB, Wright KM, Margolis SS, Marks JR, Deshmukh M, et al. Enhanced sensitivity to cytochrome c-induced apoptosis mediated by PHAPI in breast cancer cells. Cancer Res. 2006; 66(4):22102218.

26. Liu GQ, Zhang KC. Mechanisms of the anticancer action of Ganoderma lucidum (Leyss. ex Fr.) karst.: a new understanding. J. Integr. Plant Biol. 2005; 47(2):129135.

27. Bitzer J, Henkel T, Nikiforov Al, Rihner MO, Herberth MT. Developmental and reproduction toxicity studies of glycolipids from Dacryopinax spathularia. Food Chem. Toxicol. 2018; 120:430-438.

28. Wannmacher $\mathrm{L}$, Ferreira MBC. Uso de fármacos durante gestação e lactação. In: Wannmacher L, Ferreira MBC (eds.). Farmacologia Clínica para Dentistas. 1999. 2 ed., p. 270-273. Guanabara Koogan: Rio de Janeiro.

29. Christian MS. Test methods for assessing female reproductive and developmental toxicology. In: Hayes AW, ed. Principles and methods of toxicology, 2001.

30. Kang E, Cheong K-Y, Lee M-J, Kim S, Shin G-T, Kim H, et al. Severe but reversible acute kidney injury resulting from Amanita punctata poisoning. Kidney Res. Clin. Pract. 2015; 34(4):233-236.

31. Soares $A A$, Sá-Nakanishi $A B$, Bract $A$, Costa SMG, Koehnlein EA, Souza CGM, et al. Hepatoprotective effects of mushrooms. Molecules 2013; 18(7):76097630 .

32. Wu H, Dwyer KM, Fan Z, Shircore A, Fan J, Dwyer JH. Dietary fiber and progression of atherosclerosis: the Los Angeles Atherosclerosis Study. Am. J. Clin. Nutr. New York 2003; 78(6):1085-1091.

33. Londero PMG, Ribeiro ND, Cargnelutti Filho A. Teores de fibra e rendimento de grãos em populações de feijão. Ciênc. Agrotec. 2008; 32(1):167-173.
34. Kabir Y, Kimura S, Tamura T. Dietary effect of Ganoderma lucidum mushroom on blood pressure and lipid levels in spontaneously hypertensive rats (SHR). J. Nutr. Sci. Vitaminol. 1998; 34(4):433-438.

35. Mizuno T. Bioactive substances and medicinal effects of the Reishi, 2004. Disponível em: http://www.toi-reishi. com. Acesso em: 25/01/2020.

36. Assmann G, Nofer JR. Atheroprotective effects of highdensity lipoproteins. Annu. Rev. Med. 2003; 54(1):321 341.

37. Harkness SE, Wagner JE. Biologia e clínica de coelhos roedores. São Paulo, Livraria Roca, 1993.

38. Valko M, Leibfritz D, Moncol J, Cronin MTD, Mazu $\mathrm{M}$, Telser J. Free radicals and antioxidants in normal physiological functions and human disease. Int. J. Bioch. Cell Biol. 2007; 39(1):44-84.

39. Collins AR. Antioxidant intervention as a route to cancer prevention. Eur. J. Cancer 2005; 41(13):1923-1930.

40. Benzie IFF, Wachtel-Galor S. Biomarkers of long-term vegetarian diets. Adv. Clin. Chem. 2009; 47(1):169-220

41. Ferreira I, Vaz JA, Vasconcelos $\mathrm{MH}$, Martins $\mathrm{A}$. Compounds from wild mushrooms with antitumor potential. Anti-Cancer Agents Med. Chem. 2010; 10(5):424-436.

42. Li D, Sossah FL, Yang Y, Liu Z, Dai Y, Song B, et al. Genetic and pathogenic varibility of Mycogone perniciosa isolates causing wet bubble disease on Agaricus bisporus in China. Pathogens 2019; 8(4):179.

43. Tomizawa MM, Dias ES, Assis LJ, Gomide PHO, Santos JB. Variabilidade genética de isolados do cogumelo Agaricus blazei por meio de marcadores. Ciênc. Agrotec. 2007; 31(4):1242-1249.

44. Gude NM, Roberts CT, Kalionis B, King RG. Growth and function of the normal human placenta. Thromb. Res. 2004; 114(4-5):397-407.

45. Cassina M, Salviati L, Di Gianantonio E, Clementi M. Genetic susceptibility to teratogens: state of the art. Reprod. Toxicol. 2012; 34(2):186-191.

\section{CORRESPONDÊNCIA}

Yoko Oshima-Franco

Universidade de Sorocaba, Campus Cidade Universitária

Prof. Aldo Vannucchi

Rodovia Raposo Tavares, km 92,5, 18023-000

Vila Artura, Sorocaba, SP, Brasil.

E-mail: yoko.franco@prof.uniso.br. 